Rol e of spi n- or bi t coupl ing and hybri di zat i on effects in the el ectroni $c$ structure of ultrathi $n \mathrm{Bi}$ fil ms

\begin{tabular}{|l|l|}
\hline 著者 & $\begin{array}{l}\text { H rahar a T., Nagao T., Nat suda I. , Bi hl mayer } \\
\text { G, Chul kov E. V., Kor ot eev Yu. M, Echeni que } \\
\text { P. M, Sai to M neo, Hasegawa S. }\end{array}$ \\
\hline $\begin{array}{l}\text { j our nal or } \\
\text { publ i cat i on ti tl e }\end{array}$ & Physi cal Revi ew Let ter s \\
\hline vol une & 97 \\
\hline nunber & 14 \\
\hline page r ange & 146803 - 1- 146803-4 \\
\hline year & 2006- 01- 01 \\
\hline URL & ht t p: //hdl . handl e. net /2297/3488 \\
\hline
\end{tabular}




\title{
Role of Spin-Orbit Coupling and Hybridization Effects in the Electronic Structure of Ultrathin Bi Films
}

\author{
T. Hirahara, ${ }^{1}$ T. Nagao, ${ }^{2, *}$ I. Matsuda, ${ }^{1}$ G. Bihlmayer, ${ }^{3}$ E. V. Chulkov, ${ }^{4,5}$ Yu. M. Koroteev, ${ }^{4,6}$ \\ P. M. Echenique, ${ }^{4,5}$ M. Saito, ${ }^{7}$ and S. Hasegawa ${ }^{1}$ \\ ${ }^{1}$ Department of Physics, University of Tokyo, 7-3-1 Hongo, Bunkyo-ku, Tokyo 113-0033, Japan \\ ${ }^{2}$ Nano System Functionality Center, National Institute for Materials Science, 1-1 Namiki, Tsukuba, Ibaraki 305-0044, Japan \\ ${ }^{3}$ Institut für Festkörperforschung, Forschungszentrum Jülich, D-52425 Jülich, Germany \\ ${ }^{4}$ Donostia International Physics Center (DIPC), 20018 San Sebastián/Donostia, Spain \\ ${ }^{5}$ Departamento de Física de Materiales, UPV/EHU, Apdo 1072, 20080 San Sebastián, Spain \\ ${ }^{6}$ Institute of Strength Physics and Materials Science, RAS, 634021, Tomsk, Russia \\ ${ }^{7}$ Graduate School of Natural Science and Technology, Kanazawa University, Kanazawa 920-1192, Japan
}

(Received 20 December 2005; published 3 October 2006)

\begin{abstract}
The electronic structure of $\mathrm{Bi}(001)$ ultrathin films (thickness $\geq 7$ bilayers) on $\mathrm{Si}(111)-7 \times 7$ was studied by angle-resolved photoemission spectroscopy and first-principles calculations. In contrast with the semimetallic nature of bulk $\mathrm{Bi}$, both the experiment and theory demonstrate the metallic character of the films with the Fermi surface formed by spin-orbit-split surface states (SSs) showing little thickness dependence. Below the Fermi level, we clearly detected quantum well states (QWSs) at the $\bar{M}$ point, which were surprisingly found to be non-spin-orbit split; the films are "electronically symmetric" despite the obvious structural nonequivalence of the top and bottom interfaces. We found that the SSs hybridize with the QWSs near $\bar{M}$ and lose their spin-orbit-split character.
\end{abstract}

PACS numbers: 73.20. $-\mathrm{r}, 68.35 .-\mathrm{p}, 71.18 .+\mathrm{y}, 79.60 .-\mathrm{i}$

Semimetal bismuth (Bi) is one of the most extensively studied elements in solid state physics because of its extreme physical properties, such as the highest resistivity and Hall coefficient of all metals. Bi has tiny hole and electron pockets at $T$ and $L$ points, respectively, [Fig. 1(a) and $1(\mathrm{~b})]$, and therefore the Fermi wavelength $\lambda_{F}$ is very large (about $30 \mathrm{~nm}$ ). Because of this large $\lambda_{F}$, nanosized objects of $\mathrm{Bi}$ in the range of several tens of nanometers have been examined extensively toward the development of quantum-size-effect-based devices [1-3]. For example, the oscillation of the film resistance with the film thickness $d$ was reported [4] and, furthermore, it was predicted that when the lowest quantized subband of the electron pocket is raised to an energy higher than the highest hole subband, a band gap will develop [semimetal-to-semiconductor (SMSC) transition at $d \approx 30 \mathrm{~nm}$, Fig. 1(c)] [1,5]. Such a picture is based on the bulk band structure, but when the system downsizes to nanometer scale, significant contributions from the surface effects will make the system even more intriguing. In fact, surface states (SSs) on "cleaved" semi-infinite Bi surfaces have been found recently to show high electron density and large spin-orbit (SO) splitting due to the loss of the inversion symmetry (Rashba effect) $[6,7]$. The latter is a consequence of the strong SOcoupling effect in $\mathrm{Bi}$, which can also be found in the large atomic $p_{3 / 2}-p_{1 / 2}$ splitting $(1.5 \mathrm{eV})$. For the case of "ultrathin" Bi films $\left(d \ll \lambda_{F}\right)$, interaction at the substrate-film interface is naturally expected to produce profound changes in the SO splitting of both the surface and quantum well states.
In the present Letter, we report on the angle-resolved photoemission (ARPES) study of ultrathin films of $\mathrm{Bi}(001)$ grown on $\mathrm{Si}(111)-7 \times 7$ with the thicknesses from 7 bilayers up to a few nanometers $\left(d \ll \lambda_{F}\right)$. Thinner films are formed in a different allotrope structure studied in Ref. [8]. In remarkable contrast to the predicted SMSC transition, we find that the films are highly metallic and their Fermi surfaces have little thickness dependence, formed by SOsplit surface states. On the other hand, the number of states rapidly increases with the film thickness at $\bar{M}$ below $E_{F}$, which can be clearly identified as quantum well states (QWSs). Our relativistic first-principles calculation shows that these QWSs are spin degenerate in striking contrast to the strong SO-split surface-state bands [6]. This suggests that in spite of the obvious structural inequality of the top and the bottom interfaces, the electronic structure of the film behaves as if it preserves space-inversion symmetry, indicating weak substrate-film interaction. We further clarify that the hybridization of the QWSs with the SSs makes the SS bands QWS-like near the $\bar{M}$ point and lose their spin-split property.

Historically, Bi has been described in many ways of crystal indexing [9]. Here we adopt the hexagonal indexing which is mathematically the simplest and the most convenient for diffraction experiments [8]. Namely, the [001] trigonal axis in our indexing corresponds to the [111] axis in the rhombohedral notation used in previous ARPES studies [6,10] [Fig. 1(a)]. Our ARPES experiments were performed in UHV at $\sim 130 \mathrm{~K}$ with a commercial electron spectrometer (Gammadata Scienta SES-100) using unpo- 


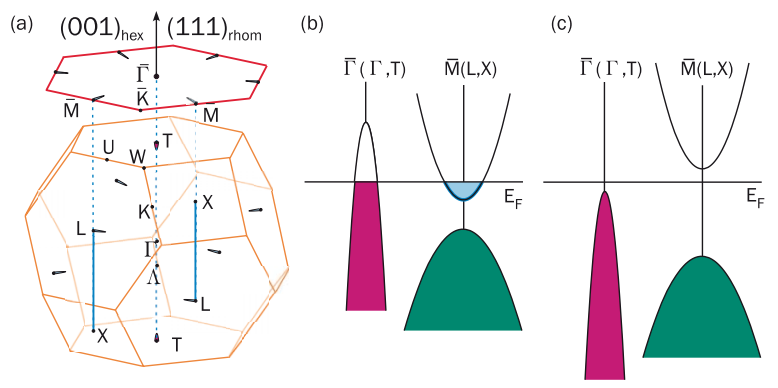

FIG. 1 (color). (a) The Fermi surface of bulk Bi depicted in bulk (orange) and its projection to the surface (red) Brillouin zone of $\mathrm{Bi}(001)_{\text {hex }}$, or $(111)_{\text {rhom. Electron (hole) pockets are }}$ filled with light blue (purple). The size of the pockets are scaled by a factor of 3. (b), (c) Schematic drawing of the Bi bulk band projection near the Fermi level $E_{F}$ before (b) and after (c) the SMSC transition as predicted in Ref. [5].

larized $\mathrm{HeI} \alpha(21.2 \mathrm{eV})$ radiation. An $n$-type ( $P$-doped, $1-10 \Omega \cdot \mathrm{cm}) \mathrm{Si}(111)$ wafer was used as the substrate. The clean $\mathrm{Si}(111)-7 \times 7$ surface was prepared by a cycle of in situ resistive heat treatments. Bi was deposited on the $7 \times 7$ surface at room temperature using a graphite effusion cell and then annealed at $\sim 350 \mathrm{~K}$. The deposition rate was $0.48 \mathrm{BL}$ (bilayer) $/ \min \left(1 \mathrm{BL}=1.14 \times 10^{15} \mathrm{~cm}^{-2}\right.$, $0.39 \mathrm{~nm}$ ) as calibrated ex situ by Rutherford backscattering spectroscopy. The overlayers prepared in this way show perfect crystallinity and abrupt interfaces as confirmed by low-energy electron diffraction (LEED), scanning tunneling microscopy (STM), and cross-sectional transmission electron microscopy (XTEM) [11,12].

The calculations have been performed using the fullpotential linearized augmented plane wave method in film geometry as implemented in the FLEUR program and local density approximation for the description of exchangecorrelation potential (for details see Ref. [6]).

Figs. 2(a)-2(c) show the photoemission intensity distribution at the Fermi level for $6.8,10$, and $17 \mathrm{BL} \mathrm{Bi(001)}$ films, respectively. The intensity increases linearly from black (minimum) to dark blue (maximum). Figure 2(d) shows the schematic drawing of the Fermi surface of the ultrathin films in the surface Brillouin zone (SBZ). There is a hexagonal electron pocket around the $\bar{\Gamma}$ point and six hole lobes along the $\bar{\Gamma}-\bar{M}$ direction. It is evident that these features are completely different from the projected bulk Fermi surface shown in Fig. 1(a) and show strong metallicity, contrary to the prediction of the SMSC transition. Also, they have hardly any thickness dependence and possess sixfold symmetry. This means that they are formed by surface states having strong two-dimensional character $[6,10]$. In fact, these features are nearly identical to the so called "crown shape" Fermi surface reported on the cleaved surface [10]. We can also find needlelike features towards the $\bar{M}$ point which correspond to the electron pocket around $\bar{M}$. This state is rather weak in its intensity and shows a slight thickness dependence of $k_{F}$. It also overlaps with the projection of the bulk electron pocket
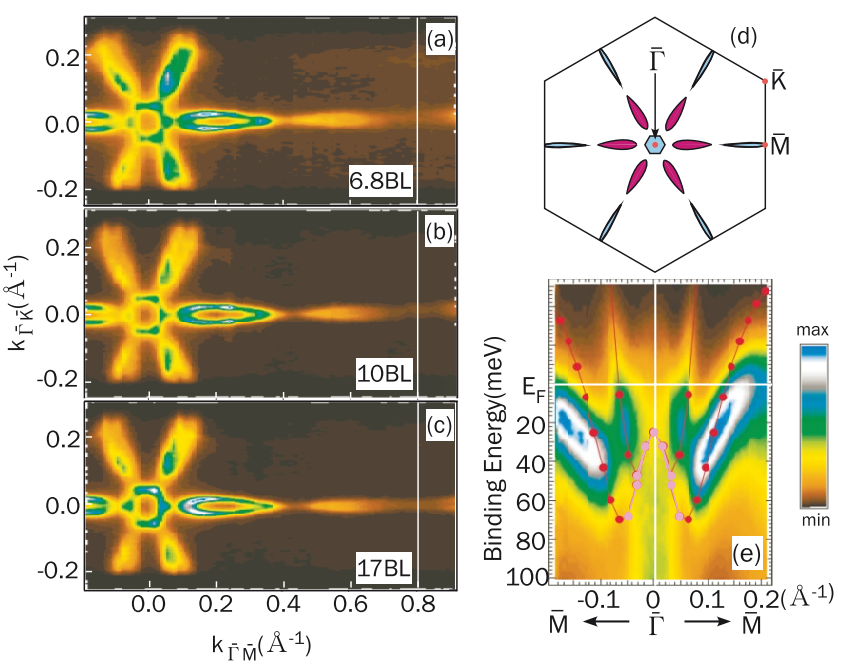

FIG. 2 (color). The Fermi surfaces of ultrathin $\mathrm{Bi}(001)$ films for 6.8 (a), 10 (b), and $17 \mathrm{BL}$ (c) films. (d) The schematic drawing of the Fermi surface of ultrathin $\mathrm{Bi}(001)$ films in the SBZ. Electron pockets are colored in light blue and hole pockets in purple. (e) The close up of the band dispersion image near the $\bar{\Gamma}$ point in the vicinity of the Fermi level along the $\bar{\Gamma}-\bar{M}$ direction for the $10 \mathrm{BL}$ film. The solid lines through the dots are the results of the first-principles calculation with one side of the film terminated with $\mathrm{H}$. Red circles are surface states and pink ones surface resonances.

suggesting that it is a resonance state (hybrid state formed by the surface state and the QWS, as shown later).

Figure 2(e) shows a close-up of the band dispersion image of this surface state near the $\bar{\Gamma}$ point in the vicinity of the Fermi level along the $\bar{\Gamma}-\bar{M}$ direction for the $10 \mathrm{BL}$ film. The basic features are the same for other thicknesses. The band dispersion is very similar to the one measured for a cleaved $\mathrm{Bi}(001)$ surface with a different photon energy [6]. The solid lines through the dots are the result of the first-principles calculation which simulates the semiinfinite $\mathrm{Bi}(001)$ crystal and shows a $k$-dependent Rashba type SO splitting of the surface-state bands. The splitting is caused by the SO-coupling Hamiltonian term,

$$
H_{\mathrm{soc}}=\frac{\hbar}{4 m_{e}^{2} c^{2}} \vec{\sigma} \cdot(\nabla V \times \vec{p}),
$$

where $\vec{\sigma}$ is the spin, $V$ represents the one-electron potential, and $\vec{p}$ is the momentum [13]. Usually in the bulk, the time-reversal symmetry $[E(\vec{k}, \uparrow)=E(-\vec{k}, \downarrow)]$ combined with the space-inversion symmetry $[E(\vec{k}, \uparrow)=E(-\vec{k}, \uparrow)]$ makes every band spin degenerate. At the crystal surface, on the other hand, the degeneracy will be lifted due to the loss of the space-inversion symmetry; each of the SO-split bands will carry only one electron $[6,14]$. Indeed, the good match between the calculated and experimental bands in Fig. 2(e) indicates that these surface states of the $\mathrm{Bi}(001)$ films have a Rashba type SO splitting [6].

Figure 3(a) shows the valence band dispersion image along the $\bar{\Gamma}-\bar{M}$ line for the $10 \mathrm{BL}$ film. To enhance spectral 


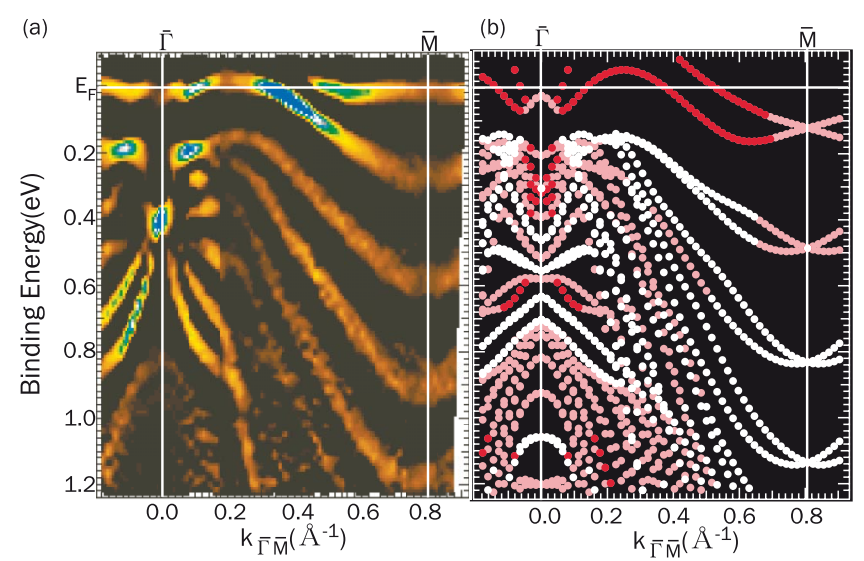

FIG. 3 (color). (a) The band structure of a $10 \mathrm{BL} \mathrm{Bi(001)} \mathrm{film}$ for the $\bar{\Gamma}-\bar{M}$ direction. (b) Calculated band dispersion for the $10 \mathrm{BL} \mathrm{Bi}$ film with one side of the film $\mathrm{H}$ terminated. Red represents surface states, pink the states close to the surface, and white the states located inside the film.

features, the image represents the second derivatives of the original ARPES spectra. We have compared the images with the raw spectra and confirmed that the peak positions agree with each other. Figure 3(b) shows the result of the first-principles calculation for a $10 \mathrm{BL}$ Bi slab with one side of the film $\mathrm{H}$ terminated. Red, pink, and white circles represent surface states, states close to the surface, and the rest, respectively. The features near $E_{F}$ in (a) are well reproduced with the calculated $k_{F}$ values showing consistency with the experiment. Near $\bar{\Gamma}$, the match is particularly good [Fig. 2(e)]. On the other hand, this calculation fails to reproduce the bands below $E_{F}$, especially the states near the $\bar{M}$ point. As was mentioned above, the calculated bands are fully SO split, except for the $\bar{\Gamma}$ and $\bar{M}$ points where the spin-split branches cross. However, as can be easily recognized from Fig. 3(a), in the photoemission spectra we find no band crossing at $\bar{M}$, which strongly suggests these bands are spin degenerate. This is surprising since even in a film as thin as $10 \mathrm{BL}$, the observed states below $E_{F}$ around $\bar{M}$ do not seem to be affected at all by the breakdown of the inversion symmetry.

To gain more insight in the relationship between the symmetry and the degeneracy, we have performed firstprinciples calculations for freestanding Bi films without terminating either side of the film with foreign atoms, assuming space-inversion symmetry of the entire film. The bands calculated in this way are spin degenerate. Figures 4(a)-4(c) show the calculated results together with the measured band dispersion for 7 (6.8 for the experiment), 10, and $17 \mathrm{BL}$ films, respectively, near the $\bar{M}$ point. By comparing Figs. 3(b) and 4(b), the preservation or the loss of the space-inversion symmetry has a great influence on the band structure, especially on the degeneracy (crossing) at $\bar{M}$. It is clear that the symmetric calculation is consistent with the overall experimental band dispersion reflecting the symmetry of the whole film, ex-
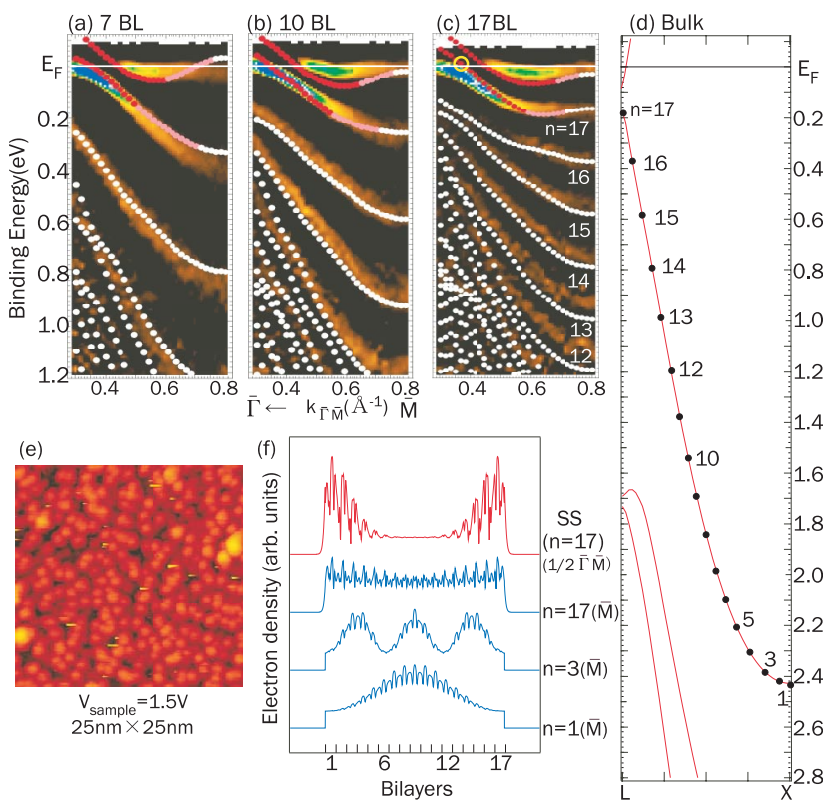

FIG. 4 (color). Calculated band structure for freestanding $\mathrm{Bi}(001)$ films assuming space-inversion symmetry for 7 (a), 10 (b), and 17 (c) BL Bi(001) films together with the measured band dispersion [6.8 BL for the experimental data of (a)] along the $\bar{\Gamma}-\bar{M}$ direction for $k>0.3 \AA^{-1}$. For the two uppermost bands close to $E_{F}$, the states whose electron density is localized at the surface are represented in red, and those localized weaker at the surface are shown in pink. The states localized inside the film are shown in white. The yellow circle in (c) is used in (f). (d) The bulk band dispersion along the $L-X$ direction and the calculated positions of QWS for the $17 \mathrm{BL}$ film. (e) STM image of the rugged wetting layer at the $\mathrm{Bi}$-Si interface taken after peeling off the Bi film. (f) The layer-resolved electron density (17 BL film) for the surface state [yellow circle in (c), the same band as $n=$ 17] and those for QWSs $(n=1,3,17)$ at $\bar{M}$.

cept for the SO-split surface states crossing $E_{F}$ near $\bar{\Gamma}$ [15]. The states located at $\bar{M}$ are particularly well reproduced. Unexpectedly, this shows that electrons in our ultrathin films really behave as if the space-inversion symmetry is preserved even though the two interfaces are asymmetric as one side is vacuum and the other side is silicon.

Another feature that we notice by comparing Figs. 4(a)4(c) is that the number of states rapidly increases as the thickness increases. Apart from the band closest to $E_{F}$ which corresponds to the surface resonance described previously, there are two, four, and six states at the $\bar{M}$ point in (a), (b), (c), respectively, in the energy range shown. These spectral features are typically found for QWSs in thin metal films [16] and the present result is the first direct observation of the QWSs for Bi films. For the 17 BL film, our calculated QWS energies being highly consistent with the experimental data are explained as quantization of the degenerate bulk band along the $L-X$ direction [17]. This is clearly understood by comparing Fig. 4(c) with Fig. 4(d) for states from $n=12$ to 17 . This fact evidences that the QWSs indeed have spin-degenerate character as should be 
in the case of films which possess space-inversion symmetry. Furthermore this indicates that the interaction between $\mathrm{Si}$ and $\mathrm{Bi}$ is very weak, and the films may actually be nearly "freestanding". In fact, in our STM observation, the films are often peeled off during the observation, which points to a very weak cohesion of the film. After the peeling off, a rugged interface structure is identified [Fig. 4(e)] and for such an atomically rugged interface the net chemical contact (interfacial bond density) between the substrate and the single-crystalline overlayer must be rather minute compared to the coherent contact between two perfectly flat surfaces. Also, our XTEM and diffraction experiments showed disordered features and an incommensurate relation at the Bi-Si interface. These experimental observations provide firm basis for the "weak interaction".

The dispersions in Figs. 4(a)-4(c) show that the second uppermost band at $\bar{M}$ is a hybrid state of the surface state and the QWS. This band behaves as a pure surface state when it locates in the bulk band gap [uncolored area in Fig. 1(b)] forming the hole lobes at midpoint of $\bar{\Gamma}-\bar{M}$ and have SO-split character. But it becomes the QWS $[n=17$ in (c)] at $\bar{M}$ and spin-degenerate when it enters the bulk band region [colored area in Fig. 1(b)]. This surprising change in spin character in the identical band can be understood by considering the charge density distribution in the thickness direction of the films. Figure 4(f) shows the calculated electron density for the SS at the midpoint of $\bar{\Gamma}-\bar{M}$ [yellow circle in (c)], and those for QWSs $[n=1,3$, 17 shown in Fig. 4(d)] at $\bar{M}$, respectively, for the $17 \mathrm{BL}$ film. For the surface state, the charge is mostly distributed in the bilayers close to the surface although not completely localized due to the coupling with the QWS. For the QWSs $n=1$ and 3 , we can clearly see the nodes and antinodes of the standing waves and for $n=17$, the feature of the standing wave is not so clear, but the charge is delocalized and distributed in the whole film. Therefore, QWSs at $\bar{M}$ are insensitive to the existence of the interface and stay spin degenerate whereas for the state near $\bar{\Gamma}$, it strongly feels the loss of space-inversion symmetry at the outermost bilayers, making the bands SO-split [Fig. 2(e)]. To our knowledge, this is the first example that clearly shows the hybridization among surface states and QWSs and the corresponding change in property of the spin. It would be interesting to directly detect this change with spinresolved ARPES measurements [14,18].

Our calculation confirms that the band closest to $E_{F}$ at $\bar{M}$ is also a hybrid state of the surface state with the bulk band (QWS) forming the electron pocket at the $L$ point. Because of this hyridization, although there are some indications of this band going above $E_{F}$ at $\bar{M}(L, X)$ in the calculated result of the 7 BL film [Fig. 4(a)], the band structure as a whole remains metallic even for films much thinner than the predicted thickness of the SMSC transition.

In conclusion, our ARPES measurements on ultrathin $\mathrm{Bi}(001)$ films showed that the films are highly metallic in contrast to the scenario of the SMSC transition. They have Fermi surfaces with little thickness dependence formed by SO-split surface states. Below the Fermi level, QWSs have been observed for the first time in Bi films. These QWSs are spin degenerate because of the weak substrate-film interaction as well as their small surface charge density which make these states insensitive to the surface structural asymmetry. Because of the hybridization among the surface and quantum well states, the surface-state bands become QWS-like near the $\bar{M}$ point and lose their spin-split character.

This work has been supported by Grants-In-Aid from the Japanese Society for the Promotion of Science, University of the Basque country, and ICORP-JST.

*Electronic address: NAGAO.Tadaaki@nims.go.jp

[1] C. A. Hoffman et al., Phys. Rev. B 48, 11431 (1993).

[2] Z. Zhang et al., Phys. Rev. B 61, 4850 (2000).

[3] T. E. Huber et al., Appl. Phys. Lett. 84, 1326 (2004).

[4] Yu. F. Ogrin et al., JETP Lett. 3, 71 (1966).

[5] V. B. Sandomirskii, Sov. Phys. JETP 25, 101 (1967).

[6] Yu. Koroteev et al., Phys. Rev. Lett. 93, 046403 (2004).

[7] J. I. Pascual et al., Phys. Rev. Lett. 93, 196802 (2004).

[8] T. Nagao et al., Phys. Rev. Lett. 93, 105501 (2004).

[9] F. Jona, Surf. Sci. 8, 57 (1967).

[10] C. R. Ast and H. Höchst, Phys. Rev. Lett. 87, 177602 (2001); 90, 016403 (2003); Phys. Rev. B 67, 113102 (2003).

[11] S. Yaginuma et al., Surf. Sci. 547, L877 (2003).

[12] T. Nagao et al., Surf. Sci. 590, L247 (2005).

[13] C. Kittel, Quantum Theory of Solids (John Wiley \& Sons, New York, 1987).

[14] M. Hoesch et al., Phys. Rev. B 69, 241401(R) (2004).

[15] There are slight discrepancies between the experimental and calculated Fermi surface near $\bar{\Gamma}$ although the general feature is quite similar.

[16] T.-C. Chiang, Surf. Sci. Rep. 39, 181 (2000).

[17] On the contrary, in the range of our interest the bulk band structure along the $\Gamma-T$ direction contains multiple bands and its dispersion is rather flat compared to that of the $L-X$ line [see Y. Liu and R. Allen, Phys. Rev. B 52, 1566 (1995)]. This makes the spacing of quantized energy levels small and likely prevents us from making clear observation of QWSs as well as the surface resonances at $\bar{\Gamma}$ [see Figs. 2(e) and 3(a)].

[18] M. Hochstrasser et al., Phys. Rev. Lett. 89, 216802 (2002). 